\title{
Quaternions and the perturbed Kepler problem
}

\section{Conference Paper}

Author(s):

Waldvogel, Jörg

Publication date:

2006-05

Permanent link:

https://doi.org/10.3929/ethz-b-000035973

Rights / license:

In Copyright - Non-Commercial Use Permitted

Originally published in:

Celestial mechanics \& dynamical astronomy 95(1-4), https://doi.org/10.1007/s10569-005-5663-7 


\title{
Quaternions and the perturbed Kepler problem
}

\author{
Jörg Waldvogel
}

Received: 3 October 2005 / Revised: 24 November 2005 /

Accepted: 5 December 2005 / Published online: 17 August 2006

(C) Springer Science+Business Media B.V. 2006

\begin{abstract}
Quaternions, introduced by Hamilton (Philos. Mag. 25, 489-495, 1844) as a generalization of complex numbers, lead to a remarkably simple representation of the perturbed three-dimensional Kepler problem as a perturbed harmonic oscillator. The paper gives an overview of this technique, including an outlook to applications in perturbation theories.
\end{abstract}

Keywords Kustaanheimo-Stiefel regularization · Quaternions ·

Perturbed Kepler problem · Birkhoff transformation

\section{Introduction}

A large branch of Celestial Mechanics is concerned with handling perturbations of the Kepler problem, described by a strongly non-linear differential equation for the vector $x \in \mathbb{R}^{n}, n=2,3$ as a function of time $t$,

$$
\ddot{x}+\mu \frac{x}{r^{3}}=\varepsilon f(x, t), \quad r=\|x\|,
$$

where $x$ is the position vector of the moving particle with respect to the central body (with gravitational parameter $\mu>0$ ), dots denote derivatives with respect to $t$, and $\varepsilon f(x, t)$ is a given small perturbation.

As will be demonstrated in the example below, the linearity of a problem leads to formally simple perturbation theories. Fortunately, there exist sets of variables in which the Kepler problem becomes linear; these are preferred variables for treating perturbed Kepler problems.

In this paper, we will revisit two closely related sets of variables that were introduced in order to regularize the collision singularity in the Kepler problem: the variables

J. Waldvogel ( $\varangle)$

Seminar for Applied Mathematics,

Swiss Federal Institute of Technology ETH,

8092 Zurich, Switzerland

e-mail:waldvogel@sam.math.ethz.ch 
introduced by Levi-Civita (1920), in which the planar Kepler problem appears as a harmonic oscillator in two dimensions, and the KS variables (Kustaanheimo 1964; Kustaanheimo and Stiefel 1965), in which the spatial Kepler problem appears as a harmonic oscillator in four dimensions. Both sets of variables have therefore the agreeable property of transforming the differential equations of the Kepler problem into a system of linear differential equations; they are therefore good variables for formulating theories of the perturbed Kepler problem. We will present a unified treatment of these two classical topics, using complex variables in the planar case and quaternions in the three-dimensional case. For completeness we mention that in a set of variables based on radial inversion in momentum space (see, e.g., Siegel and Moser 1971; or the summary in Celletti 2002) the Kepler problem also becomes linear.

The use of quaternions for the purpose of regularization of the Kepler problem in three dimensions has been contemplated before. In the comprehensive text by Stiefel and Scheifele (1971) the use of quaternions was taken into consideration (p. 286), but clearly rejected: "Any attempt to substitute the theory of the KS matrix by the more popular theory of the quaternion matrices leads to failure or at least to a very unwieldy formalism”. Almost simultaneously, Vivarelli (1994) and Vrbik (1994, 1995) demonstrated the usefulness of quaternions in this field. Here we will describe a new, elegant way of handling the three-dimensional case in complete analogy to the well-known planar case by introducing an unconventional conjugation of quaternions (see the definition in Eq. (24) below), first mentioned by Waldvogel (2006).

Perturbation theories of ordinary differential equations are comparatively simple for linear problems. Consider, e.g., the perturbed system

$$
\dot{x}(t)+A(t) x(t)-b(t)=\varepsilon f(x, t), \quad x: t \in \mathbb{R} \mapsto x(t) \in \mathbb{R}^{n}
$$

of linear differential equations, where $A(t)$ is a given time-dependent matrix. Equation (2) may formally be solved to arbitrary order by the series

$$
x(t)=x_{0}(t)+\varepsilon x_{1}(t)+\varepsilon^{2} x_{2}(t)+\cdots,
$$

where $x_{k}(t)$ satisfies the linear differential equation

$$
\dot{x_{k}}(t)+A(t) x_{k}(t)=f_{k-1}(t), \quad k=0,1,2, \ldots
$$

Here $f_{-1}(t):=b(t)$, and $f_{0}(t), f_{1}(t), \ldots$ are defined as the coefficients of the formal Taylor series of $f(x, t)$ with respect to $\varepsilon$ :

$$
\sum_{k=0}^{\infty} \varepsilon^{k} f_{k}(t)=f\left(x_{0}(t)+\varepsilon x_{1}(t)+\varepsilon^{2} x_{2}(t)+\cdots, t\right) .
$$

Note that the linear differential equations (3) are all of the type of the unperturbed problem $k=0$; they only differ in their right-hand sides.

In Sect. 2, Levi-Civita's regularization procedure in complex notation will be summarized. Section 3 contains a brief introduction to quaternion algebra and states the KS transformation in quaternion notation. In Sect. 4, the main part of this paper, we develop a simple, concise way of transforming the spatial perturbed Kepler problem into a perturbed harmonic oscillator in four dimensions. As a byproduct, an elegant representation of the spatial Birkhoff transformation in quaternion notation will be given in Sect. 5. 


\section{The Levi-Civita transformation}

Here we summarize the three steps necessary for regularizing (and thus "linearizing") the perturbed planar Kepler problem by Levi-Civita's transformation. Throughout this section, we use complex notation, i.e. instead of the vectors $x=\left(x_{1}, x_{2}\right)^{T} \in \mathbb{R}^{2}, f=$ $\left(f_{1}, f_{2}\right)^{T}$ we use the corresponding complex numbers $\mathbf{x}=x_{1}+i x_{2} \in \mathbb{C}, \mathbf{f}=f_{1}+i f_{2} \in \mathbb{C}$.

\subsection{First step: slow-motion movie}

Instead of the physical time $t$ a new independent variable $\tau$, called the fictitious time, is introduced by the differential relation

$$
\mathrm{d} t=r \cdot \mathrm{d} \tau, \quad \frac{\mathrm{d}}{\mathrm{d} \tau}\left(\mathrm{)}=()^{\prime} .\right.
$$

Therefore, the ratio $\mathrm{d} t / \mathrm{d} \tau$ of two infinitesimal increments is made proportional to the distance $r$; the movie is run in slow-motion whenever $r$ becomes small. With the differentiation rules

$$
\frac{\mathrm{d}}{\mathrm{d} t}=\frac{1}{r} \frac{\mathrm{d}}{\mathrm{d} \tau}, \quad \frac{\mathrm{d}^{2}}{\mathrm{~d} t^{2}}=\frac{1}{r^{2}} \frac{\mathrm{d}^{2}}{\mathrm{~d} \tau^{2}}-\frac{r^{\prime}}{r^{3}} \frac{\mathrm{d}}{\mathrm{d} \tau} .
$$

Equation (1) transforms into

$$
r \mathbf{x}^{\prime \prime}-r^{\prime} \mathbf{x}^{\prime}+\mu \mathbf{x}=r^{3} \varepsilon \mathbf{f} \in \mathbb{C} .
$$

\subsection{Second step: conformal squaring}

This part of Levi-Civita's regularization procedure consists of representing the complex physical coordinate $\mathbf{x}$ as the square $\mathbf{u}^{2}$ of a complex variable $\mathbf{u}=u_{1}+i u_{2} \in \mathbb{C}$,

$$
\mathbf{x}=\mathbf{u}^{2},
$$

i.e. the mapping from the parametric plane to the physical plane is chosen as a conformal squaring. This is based on the observation that conformal squaring maps an origin-centered ellipse to a Keplerian ellipse with one focus at the origin (see Fig. 1). Equation (6) implies

$$
r=|\mathbf{x}|=|\mathbf{u}|^{2}=\mathbf{u} \overline{\mathbf{u}},
$$

and differentiation of Eqs. (6) and (7) yields

$$
\mathbf{x}^{\prime}=2 \mathbf{u} \mathbf{u}^{\prime}, \quad \mathbf{x}^{\prime \prime}=2\left(\mathbf{u} \mathbf{u}^{\prime \prime}+\mathbf{u}^{\prime 2}\right) \in \mathbb{C}, \quad r^{\prime}=\mathbf{u}^{\prime} \overline{\mathbf{u}}+\mathbf{u} \overline{\mathbf{u}^{\prime}} .
$$

By substituting this into (5), cancelling two equal terms $\left(2 r \mathbf{u}^{\prime 2}\right.$ and $\left.2 \mathbf{u}^{\prime} \mathbf{u} \mathbf{u} \mathbf{u}^{\prime}\right)$ and dividing by $\mathbf{u}$ we obtain

$$
2 r \mathbf{u}^{\prime \prime}+\left(\mu-2\left|\mathbf{u}^{\prime}\right|^{2}\right) \mathbf{u}=r^{2} \overline{\mathbf{u}} \varepsilon \mathbf{f} .
$$

Remark Obtaining initial values $\mathbf{u}(0)=\sqrt{\mathbf{x}(0)}$ requires the computation of a complex square root. This can conveniently be accomplished by means of the formula

$$
\sqrt{\mathbf{x}}=\frac{\mathbf{x}+|\mathbf{x}|}{\sqrt{2(|\mathbf{x}|+\operatorname{Re} \mathbf{x})}},
$$



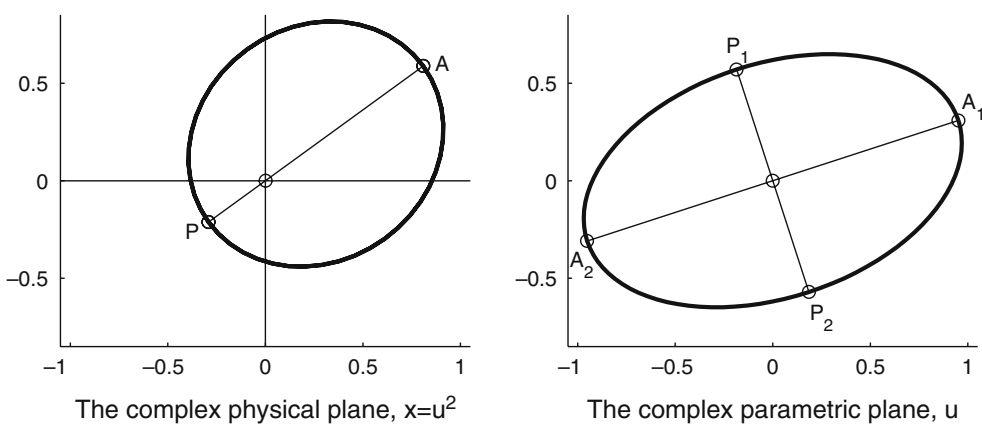

Fig. 1 The image of a (doubly covered) Keplerian ellipse with one focus at the origin of the physical plane (left) under the conformal square root is an ellipse centered at the origin of the parametric plane (right)

which reflects the observation that the complex vector $\sqrt{\mathbf{x}}$ has the direction of the bisector between $\mathbf{x}$ and the real vector $|\mathbf{x}|$; it holds in the range $-\pi<\arg (\mathbf{x})<\pi$. The alternative formula

$$
\sqrt{\mathbf{x}}=\frac{\mathbf{x}-|\mathbf{x}|}{i \sqrt{2(|\mathbf{x}|-\operatorname{Re} \mathbf{x})}}
$$

holds in $0<\arg (\mathbf{x})<2 \pi$ and agrees with (10) in the upper half-plane; it therefore provides the analytic continuation of (10) into the sector $\pi \leqslant \arg (\mathbf{x})<2 \pi$. Furthermore, it avoids a loss of accuracy near the negative real axis $\mathbf{x}<0$.

\subsection{Third step: fixing the energy}

This step is simple for the unperturbed problem, $\varepsilon=0$. Integrating the inner product of Eq. (1) and the vector $\dot{x} \in \mathbb{R}^{2}$ yields the well-known energy equation

$$
\frac{1}{2}\|\dot{x}\|^{2}-\frac{\mu}{r}=-h=\text { const, }
$$

where the energy constant $h$ is chosen such that $h>0$ corresponds to an elliptic orbit. From (4) and (8) there follows (using again complex notation):

$$
\dot{\mathbf{x}}=\frac{1}{r} \cdot 2 \mathbf{u} \mathbf{u}^{\prime}, \quad \frac{1}{2}|\dot{\mathbf{x}}|^{2}=2 \frac{\left|\mathbf{u}^{\prime}\right|^{2}}{r},
$$

and (11) implies

$$
\mu-2\left|\mathbf{u}^{\prime}\right|^{2}=r h .
$$

Substituting this into (9) and dividing by $r$ yields

$$
2 \mathbf{u}^{\prime \prime}+h \mathbf{u}=0,
$$

a system of linear differential equations describing a harmonic oscillator in two dimensions with frequency $\omega=\sqrt{h / 2}$.

In the perturbed case $h$ of Eq. (11) is no longer a constant, but is a slowly varying function and satisfies the differential equation

$$
\dot{h}=-\langle\dot{x}, \varepsilon f\rangle \quad \text { or } \quad h^{\prime}=-\left\langle x^{\prime}, \varepsilon f\right\rangle,
$$


where $\langle x, y\rangle$ denotes the inner product of the vectors $x, y \in \mathbb{R}^{2}$. The energy equation (13) still holds, and instead of (14) we obtain

$$
2 \mathbf{u}^{\prime \prime}+h \mathbf{u}=|\mathbf{u}|^{2} \overline{\mathbf{u}} \varepsilon \mathbf{f} .
$$

Remark Equation (16), together with the second Eq. (15), describes a perturbed harmonic oscillator with slowly varying frequency; it may be transformed to constant frequency by introducing a new independent variable $s$ proportional to the osculating eccentric anomaly, e.g., according to the differential relation

$$
\mathrm{d} s=\sqrt{h} \mathrm{~d} \tau .
$$

This results in the system of differential equations

$$
\frac{\mathrm{d} h}{\mathrm{~d} s}=-\frac{r}{\sqrt{h}}\langle\dot{x}, \varepsilon f\rangle, \quad 2 \frac{\mathrm{d}^{2} \mathbf{u}}{\mathrm{d} s^{2}}+\mathbf{u}=\frac{r}{h} \overline{\mathbf{u}} \varepsilon \mathbf{f}-\frac{1}{h} \frac{\mathrm{d} h}{\mathrm{~d} s} \frac{\mathrm{d} \mathbf{u}}{\mathrm{d} s}
$$

(for more details regarding applications to perturbation theories, see Waldvogel 2006).

\section{Quaternion algebra and the KS transformation}

In this section we indicate how Levi-Civita's regularization procedure may be generalized to three-dimensional motion. The essential step is to replace the conformal squaring of Sect. 2.2 by the Kustaanheimo-Stiefel (KS) transformation. A preliminary version of this transformation using spinor notation was proposed by Kustaanheimo (1964); the full theory was developed in a subsequent joint paper (Kustaanheimo and Stiefel 1965); the entire topic is extensively discussed in the comprehensive text by Stiefel and Scheifele (1971). The relevant mapping from the 3-sphere onto the 2-sphere was discovered already by Hopf (1931) and is referred to in topology as the Hopf mapping.

Both the Levi-Civita and the KS regularization share the property of "linearizing" the equations of motion of the two-body problem. Quaternion algebra, introduced by Hamilton (1844), turns out to be very well suited as a tool for regularizing the three-dimensional Kepler motion, as was observed by Vivarelli (1994) and Vrbik (1994, 1995). Here we will present a new elegant way of extending the Levi-Civita regularization to three dimensions by means of quaternions.

\subsection{Basics}

Quaternion algebra is a generalization of the algebra of complex numbers obtained by using three independent "imaginary" units $i, j, k$. As for the single imaginary unit $i$ in the algebra of complex numbers, the rules

$$
i^{2}=j^{2}=k^{2}=-1
$$

are postulated, together with the non-commutative multiplication rules

$$
i j=-j i=k, \quad j k=-k j=i, \quad k i=-i k=j .
$$

Given the real numbers $u_{l} \in \mathbb{R}, l=0,1,2,3$, the object

$$
\mathbf{u}=u_{0}+i u_{1}+j u_{2}+k u_{3}
$$


is called a quaternion $\mathbf{u} \in \mathbb{U}$, where $\mathbb{U}$ denotes the set of all quaternions (in the remaining sections bold-face characters denote quaternions). The sum $i u_{1}+j u_{2}+k u_{3}$ is called the quaternion part of $\mathbf{u}$, whereas $u_{0}$ is naturally referred to as its real part. The above multiplication rules and vector space addition define the quaternion algebra. Multiplication is generally non-commutative; however, any quaternion commutes with a real:

$$
c \mathbf{u}=\mathbf{u} c, \quad c \in \mathbb{R}, \quad \mathbf{u} \in \mathbb{U},
$$

and for any three quaternions $\mathbf{u}, \mathbf{v}, \mathbf{w} \in \mathbb{U}$ the associative law holds:

$$
(\mathbf{u} \mathbf{v}) \mathbf{w}=\mathbf{u}(\mathbf{v} \mathbf{w}) .
$$

The quaternion $\mathbf{u}$ may naturally be associated with the corresponding vector $u=$ $\left(u_{0}, u_{1}, u_{2}, u_{3}\right) \in \mathbb{R}^{4}$. For later reference we introduce notation for three-vectors in two important particular cases: $\vec{u}=\left(u_{1}, u_{2}, u_{3}\right) \in \mathbb{R}^{3}$ for the vector associated with the pure quaternion $\mathbf{u}=i u_{1}+j u_{2}+k u_{3}$, and $\underline{u}=\left(u_{0}, u_{1}, u_{2}\right)$ for the vector associated with the quaternion with a vanishing $k$-component, $\mathbf{u}=u_{0}+i u_{1}+j u_{2}$.

For convenience we also introduce the vector $\vec{l}=(i, j, k)$; the quaternion $\mathbf{u}$ may then be written formally as $\mathbf{u}=u_{0}+\langle\vec{\imath}, \vec{u}\rangle$. For the two quaternion products of $\mathbf{u}$ and $\mathbf{v}=v_{0}+\langle\vec{\imath}, \vec{v}\rangle$, we then obtain the concise expressions

$$
\begin{aligned}
& \mathbf{u} \mathbf{v}=u_{0} v_{0}-\langle\vec{u}, \vec{v}\rangle+\left\langle\vec{\imath}, u_{0} \vec{v}+v_{0} \vec{u}+\vec{u} \times \vec{v}\right\rangle, \\
& \mathbf{v} \mathbf{u}=u_{0} v_{0}-\langle\vec{u}, \vec{v}\rangle+\left\langle\vec{\imath}, u_{0} \vec{v}+v_{0} \vec{u}-\vec{u} \times \vec{v}\right\rangle,
\end{aligned}
$$

where $x$ denotes the vector product. Note that the non-commutativity shows only in the sign of the term with the vector product.

The conjugate $\overline{\mathbf{u}}$ of the quaternion $\mathbf{u}$ is defined as

$$
\overline{\mathbf{u}}=u_{0}-i u_{1}-j u_{2}-k u_{3} ;
$$

then the modulus $|\mathbf{u}|$ of $\mathbf{u}$ is obtained from

$$
|\mathbf{u}|^{2}=\mathbf{u} \overline{\mathbf{u}}=\overline{\mathbf{u}} \mathbf{u}=\sum_{l=0}^{3} u_{l}^{2} .
$$

As transposition of a product of matrices, conjugation of a quaternion product reverses the order of its factors:

$$
\overline{\mathbf{u} \mathbf{v}}=\overline{\mathbf{v}} \overline{\mathbf{u}} .
$$

\subsection{The KS transformation with quaternions}

Here, we will revisit KS regularization and present a new, elegant derivation of it, using quaternion algebra and an unconventional "conjugate" $\mathbf{u}^{\star}$ referred to as the star conjugate of the quaternion $\mathbf{u}=u_{0}+i u_{1}+j u_{2}+k u_{3}$ :

$$
\mathbf{u}^{\star}:=u_{0}+i u_{1}+j u_{2}-k u_{3} .
$$

The star conjugate of $\mathbf{u}$ may be expressed in terms of the conventional conjugate $\overline{\mathbf{u}}$ as

$$
\mathbf{u}^{\star}=k \overline{\mathbf{u}} k^{-1}=-k \overline{\mathbf{u}} k
$$

Springer 
however, it turns out that the definition (24) leads to a particularly elegant treatment of KS regularization. The following elementary properties are easily verified:

$$
\begin{aligned}
\left(\mathbf{u}^{\star}\right)^{\star} & =\mathbf{u}, \\
\left|\mathbf{u}^{\star}\right|^{2} & =|\mathbf{u}|^{2}, \\
(\mathbf{u ~ v})^{\star} & =\mathbf{v}^{\star} \mathbf{u}^{\star} .
\end{aligned}
$$

Consider now the mapping

$$
\mathbf{u} \in \mathbb{U} \longmapsto \mathbf{x}=\mathbf{u} \mathbf{u}^{\star} .
$$

Star conjugation immediately yields $\mathbf{x}^{\star}=\left(\mathbf{u}^{\star}\right)^{\star} \mathbf{u}^{\star}=\mathbf{x}$; hence $\mathbf{x}$ is a quaternion of the form $\mathbf{x}=x_{0}+i x_{1}+j x_{2}$ which may be associated with the vector $\underline{x}=\left(x_{0}, x_{1}, x_{2}\right) \in \mathbb{R}^{3}$. From $\mathbf{u}=u_{0}+i u_{1}+j u_{2}+k u_{3}$ we obtain

$$
\begin{aligned}
& x_{0}=u_{0}^{2}-u_{1}^{2}-u_{2}^{2}+u_{3}^{2}, \\
& x_{1}=2\left(u_{0} u_{1}-u_{2} u_{3}\right), \\
& x_{2}=2\left(u_{0} u_{2}+u_{1} u_{3}\right),
\end{aligned}
$$

which is exactly the KS transformation in its classical form or - up to a permutation of the indices - the Hopf map. Therefore, we have

Theorem 1 The KS transformation which maps $u=\left(u_{0}, u_{1}, u_{2}, u_{3}\right) \in \mathbb{R}^{4}$ to $\underline{x}=$ $\left(x_{0}, x_{1}, x_{2}\right) \in \mathbb{R}^{3}$ is given by the quaternion relation

$$
\mathbf{x}=\mathbf{u} \mathbf{u}^{\star}
$$

where $\mathbf{u}=u_{0}+i u_{1}+j u_{2}+k u_{3}, \mathbf{x}=x_{0}+i x_{1}+j x_{2}$.

Corollary 1 The norms of the vectors $\underline{x}$ and $u$ satisfy

$$
r:=\|\underline{x}\|=\|u\|^{2}=\mathbf{u} \overline{\mathbf{u}} .
$$

Proof By appropriately combining the two conjugations and using the rules (18), (19), (22), (23) and (25) we obtain

$$
\|\underline{x}\|^{2}=\mathbf{x} \overline{\mathbf{x}}=\mathbf{u}\left(\mathbf{u}^{\star} \overline{\mathbf{u}}^{\star}\right) \overline{\mathbf{u}}=\left|\mathbf{u}^{\star}\right|^{2}|\mathbf{u}|^{2}=|\mathbf{u}|^{4}=\|u\|^{4}
$$

from where the statement follows.

\subsection{The inverse map}

Since the mapping (27) does not preserve the dimension its inverse in the usual sense does not exist. However, the present quaternion formalism yields an elegant way of finding the corresponding fibration of the original space $\mathbb{R}^{4}$. Being given a quaternion $\mathbf{x}=x_{0}+i x_{1}+j x_{2}$ with vanishing $k$-component, $\mathbf{x}=\mathbf{x}^{\star}$, we want to find all quaternions $\mathbf{u}$ such that $\mathbf{u} \mathbf{u}^{\star}=\mathbf{x}$. We propose the following solution in two steps:

First step: Find a particular solution $\mathbf{u}=\mathbf{v}=\mathbf{v}^{\star}=v_{0}+i v_{1}+j v_{2}$ which has also a vanishing $k$-component. Since $\mathbf{v} \mathbf{v}^{\star}=\mathbf{v}^{2}$ we may use Eq. (10), which was developed for the complex square root, also for the square root of a quaternion:

$$
\mathbf{v}=\frac{\mathbf{x}+|\mathbf{x}|}{\sqrt{2\left(|\mathbf{x}|+x_{0}\right)}} .
$$

Clearly, v has a vanishing $k$-component. 
Second step: The entire family of solutions (the fiber corresponding to $\mathbf{x}$, geometrically a circle in $\mathbb{R}^{4}$ parametrized by the angle $\varphi$ ), is given by

$$
\mathbf{u}=\mathbf{v} \cdot e^{k \varphi}=\mathbf{v}(\cos \varphi+k \sin \varphi) .
$$

Proof $\mathbf{u} \mathbf{u}^{\star}=\mathbf{v} e^{k \varphi} e^{-k \varphi} \mathbf{v}^{\star}=\mathbf{v} \mathbf{v}^{\star}=\mathbf{x}$.

\section{$4 \mathrm{KS}$ regularization with quaternions}

In order to regularize the perturbed three-dimensional Kepler motion by means of the KS transformation it is necessary to look at the properties of the map (26) under differentiation.

The transformation (26) or (27) is a mapping from $\mathbb{R}^{4}$ to $\mathbb{R}^{3}$; it therefore, leaves one degree of freedom in the parametric space undetermined. In KS theory (Kustaanheimo and Stiefel, 1965; Stiefel and Scheifele, 1971), this freedom is taken advantage of by trying to inherit as much as possible of the conformality properties of the LeviCivita map, but other approaches exist (e.g., Vrbik 1995). By imposing the "bilinear relation"

$$
2\left(u_{3} \mathrm{~d} u_{0}-u_{2} \mathrm{~d} u_{1}+u_{1} \mathrm{~d} u_{2}-u_{0} \mathrm{~d} u_{3}\right)=0
$$

between the vector $u=\left(u_{0}, u_{1}, u_{2}, u_{3}\right)$ and its differential $\mathrm{d} u$ on orbits the tangential map of (27) becomes a linear map with an orthogonal (but non-normalized) matrix.

This property has a simple consequence on the differentiation of the quaternion representation (26) of the KS transformation. Considering the non-commutativity of the quaternion product, the differential of Eq. (26) becomes

$$
\mathrm{d} \mathbf{x}=\mathrm{d} \mathbf{u} \cdot \mathbf{u}^{\star}+\mathbf{u} \cdot \mathrm{d} \mathbf{u}^{\star}
$$

whereas (29) takes the form of a commutator relation,

$$
\mathbf{u} \cdot \mathrm{d} \mathbf{u}^{\star}-\mathrm{d} \mathbf{u} \cdot \mathbf{u}^{\star}=0 .
$$

Combining (30) with the relation (31) yields the elegant result

$$
\mathrm{d} \mathbf{x}=2 \mathbf{u} \cdot \mathrm{d} \mathbf{u}^{\star}
$$

i.e. the bilinear relation (29) of KS theory is equivalent with the requirement that the tangential map of $\mathbf{u} \mapsto \mathbf{u} \mathbf{u}^{\star}$ behaves as in a commutative algebra.

By using the tools collected in Section 3 together with Eq. (32) the regularization procedure outlined in Section 2 will now be carried out for the three-dimensional perturbed Kepler problem. Care must be taken to preserve the order of the factors in quaternion products. Exchanging two factors is permitted if one of the factors is real or if the factors are mutually conjugate. An important tool for simplifying expressions is regrouping factors of multiple products according to the associative law (19). In order to stress the simplicity of this approach, we present all the details of the formal computations. 
4.1 First step in space: slow-motion movie

Let $\mathbf{x}=x_{0}+i x_{1}+j x_{2} \in \mathbb{U}$ be the quaternion associated with the position vector $\underline{x}=\left(x_{0}, x_{1}, x_{2}\right) \in \mathbb{R}^{3}$; then the perturbed Kepler problem (1) is given by

$$
\ddot{\mathbf{x}}+\mu \frac{\mathbf{x}}{r^{3}}=\varepsilon \mathbf{f}(\mathbf{x}, t) \in \mathbb{U}, \quad r=|\mathbf{x}|,
$$

where $\mathbf{f}(\mathbf{x}, t)=f_{0}(\mathbf{x}, t)+i f_{1}(\mathbf{x}, t)+j f_{2}(\mathbf{x}, t)=\mathbf{f}^{\star}(\mathbf{x}, t)$ is the quaternion associated with the perturbation $f(\underline{x}, t) \in \mathbb{R}^{3}$.

The first transformation step calls for introducing the fictitious time $\tau$ according to Eq. (4), $d t=r \cdot d \tau$; the result is formally identical with Eq. (5),

$$
r \mathbf{x}^{\prime \prime}-r^{\prime} \mathbf{x}^{\prime}+\mu \mathbf{x}=r^{3} \varepsilon \mathbf{f} \in \mathbb{U} .
$$

4.2 Second step: KS transformation with quaternions

Instead of the conformal squaring according to Eq. (6) we use the KS transformation (26),

$$
\mathbf{x}=\mathbf{u} \mathbf{u}^{\star}, \quad r:=|\mathbf{x}|=\mathbf{u} \overline{\mathbf{u}} .
$$

Differentiation by means of the commutator relation (31) yields

$$
\mathbf{x}^{\prime}=2 \mathbf{u} \mathbf{u}^{\star^{\prime}}, \quad \mathbf{x}^{\prime \prime}=2 \mathbf{u} \mathbf{u}^{\star^{\prime \prime}}+2 \mathbf{u}^{\prime} \mathbf{u}^{\star^{\prime}}, \quad r^{\prime}=\mathbf{u}^{\prime} \overline{\mathbf{u}}+\mathbf{u} \overline{\mathbf{u}}^{\prime} .
$$

Substitution of (35) and (36) into (34) results in the lengthy equation

$$
(\mathbf{u} \overline{\mathbf{u}})\left(2 \mathbf{u} \mathbf{u}^{\star^{\prime \prime}}+2 \mathbf{u}^{\prime} \mathbf{u}^{\star^{\prime}}\right)-\left(\mathbf{u}^{\prime} \overline{\mathbf{u}}+\mathbf{u} \overline{\mathbf{u}}^{\prime}\right) 2 \mathbf{u}^{\star^{\prime}}+\mu \mathbf{u} \mathbf{u}^{\star}=r^{3} \varepsilon \mathbf{f},
$$

which is considerably simplified by observing that the second and third term - after applying the distributive law-compensate:

$$
2(\mathbf{u} \overline{\mathbf{u}}) \mathbf{u}^{\prime} \mathbf{u}^{\star^{\prime}}-2 \mathbf{u}^{\prime}(\overline{\mathbf{u}} \mathbf{u}) \mathbf{u}^{\star^{\prime}}=0 .
$$

Furthermore, by means of (18), (19) and (31) the fourth term of (37) may be simplified as follows:

$$
-2\left(\mathbf{u} \overline{\mathbf{u}}^{\prime}\right)\left(\mathbf{u} \mathbf{u}^{\star^{\prime}}\right)=-2 \mathbf{u}\left(\overline{\mathbf{u}}^{\prime} \mathbf{u}^{\prime}\right) \mathbf{u}^{\star}=-2\left|\mathbf{u}^{\prime}\right|^{2} \mathbf{u} \mathbf{u}^{\star} .
$$

By using this and left-dividing by $\mathbf{u}$ Equation (37) now becomes

$$
2 r \mathbf{u}^{\star^{\prime \prime}}+\left(\mu-2\left|\mathbf{u}^{\prime}\right|^{2}\right) \mathbf{u}^{\star}=r^{2} \overline{\mathbf{u}} \varepsilon \mathbf{f}
$$

in almost perfect formal agreement with Eq. (9) of the planar case.

4.3 Third step: fixing the energy in space

In formal agreement with the planar case the energy equation expressed by fictitious time is

$$
\frac{1}{2 r^{2}}\left|\mathbf{x}^{\prime}\right|^{2}-\frac{\mu}{r}=-h \quad \text { with } \quad h^{\prime}=-\left\langle\underline{\underline{\prime}^{\prime}}, \varepsilon \underline{f}\right\rangle .
$$

From (36), (25), (28) we have

$$
\left|\mathbf{x}^{\prime}\right|^{2}=\mathbf{x}^{\prime} \overline{\mathbf{x}}^{\prime}=4 \mathbf{u}\left(\mathbf{u}^{\star^{\prime}} \overline{\mathbf{u}}^{\star^{\prime}}\right) \overline{\mathbf{u}}=4 r\left|\mathbf{u}^{\prime}\right|^{2},
$$


and the first equation of (39) becomes

$$
\mu-2\left|\mathbf{u}^{\prime}\right|^{2}=r h
$$

in formal agreement with Eq. (13) found for the planar case. Substituting this into the star-conjugate of (38) and dividing by $r$ yields the elegant final result

$$
2 \mathbf{u}^{\prime \prime}+h \mathbf{u}=|\mathbf{u}|^{2} \varepsilon \mathbf{f} \overline{\mathbf{u}}^{\star}
$$

a differential equation in perfect agreement with (16) for the planar case; however, it takes more than an educated guess to get the correct right-hand side.

\section{The Birkhoff transformation}

The topic of this section is not directly related to the preceding text; we add it here because the quaternion tools discussed before allow for an elegant representation of the spatial Birkhoff transformation.

This regularizing transformation was proposed by Birkhoff (1915), in order to regularize all singularities of the planar restricted three-body problem with a single transformation. Half a century later Stiefel and Waldvogel (1965) published a generalization of Birkhoff's transformation to three dimensions, using the KS transformation. Later these ideas were used by Waldvogel (1967a, b).

Here, we will first revisit the classical Birkhoff transformation (the same conformal map is known in aerodynamics as the Joukowsky transformation) and represent it as the composition of three elementary conformal mappings; this will then readily generalize to the spatial situation by means of quaternions.

Consider a rotating physical plane parametrized by the complex variable $\mathbf{y} \in \mathbb{C}$; for convenience we assume the fixed primaries of the restricted three-body problem to be situated at the points $\mathrm{A}, \mathrm{C}$ given by the complex posititons $\mathbf{y}=-1$ and $\mathbf{y}=1$, respectively (see Fig. 2). The complex variable of the parametric plane will be denoted by $\mathbf{v}$ and will be normalized in such a way that the primaries are mapped to $\mathbf{v}=-1$ or $\mathbf{v}=1$, respectively.

The key observation is that Levi-Civita's conformal map (6), $\mathbf{u} \mapsto \mathbf{x}=\mathbf{u}^{2}$, not only regularizes collisions at $\mathbf{x}=0$ but also analogous singularities at $\mathbf{x}=\infty$. This is seen
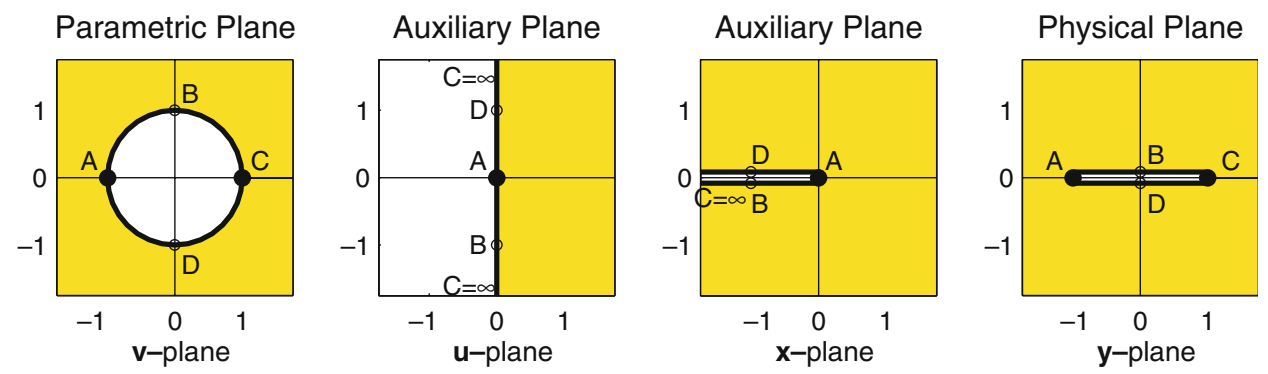

$$
\mathbf{v} \mapsto \mathbf{u}=\frac{\mathbf{v}+1}{\mathbf{v}-1} \quad \mathbf{u} \mapsto \mathbf{x}=\mathbf{u}^{2} \quad \mathbf{x} \mapsto \mathbf{y}=\frac{\mathbf{x}+1}{\mathbf{x}-1}
$$

Fig. 2 The sequence of conformal maps generating the planar Birkhoff transformation 
by closing the complex planes to become Riemann spheres (by adding the point at infinity) and using inversions $\mathbf{x}=1 / \tilde{\mathbf{x}}, \mathbf{u}=1 / \tilde{\mathbf{u}}$.

Taking advantage of this fact, we first map the $\mathbf{v}$-sphere to an auxiliary $\mathbf{u}$-sphere by the Möbius transformation

$$
\mathbf{v} \longmapsto \mathbf{u}=\frac{\mathbf{v}+1}{\mathbf{v}-1}=1+\frac{2}{\mathbf{v}-1},
$$

which takes the primaries $\mathrm{A}, \mathrm{C}$ to the points $\mathbf{u}=0, \mathbf{u}=\infty$, respectively. The LeviCivita map (6) will leave these points invariant while regularizing collisions at A or C. Finally, the Möbius transformation

$$
\mathbf{x} \longmapsto \mathbf{y}=\frac{\mathbf{x}+1}{\mathbf{x}-1}=1+\frac{2}{\mathbf{x}-1}
$$

maps A, C to $\mathbf{y}=-1$ and $\mathbf{y}=1$, respectively. The composition of the maps (42), (6), (43) yields

$$
\mathbf{y}=\frac{\left(\frac{\mathbf{v}+1}{\mathbf{v}-1}\right)^{2}+1}{\left(\frac{\mathbf{v}+1}{\mathbf{v}-1}\right)^{2}-1} \text { or } \mathbf{y}=\frac{1}{2}\left(\mathbf{v}+\frac{1}{\mathbf{v}}\right)
$$

the well-known map used by Joukowsky and Birkhoff.

In the spatial case, we choose $\mathbf{v}, \mathbf{u}, \mathbf{x}, \mathbf{y} \in \mathbb{U}$ to be quaternions, $\mathbf{x}=\mathbf{x}^{\star}, \mathbf{y}=\mathbf{y}^{\star}$ being quaternions with vanishing $k$-components associated with 3 -vectors $\underline{x}, \underline{y}$. Then the mappings (42), (43), now being shifted inversions in four or three dimensions, are both conformal maps, in fact the only conformal maps existing in those dimensions, except for the translations, magnifications, and rotations. Composing these with the KS or Hopf map (35), $\mathbf{u} \mapsto \mathbf{x}=\mathbf{u} \mathbf{u}^{\star}$, yields

$$
\mathbf{y}=1+\left(\mathbf{v}^{\star}-1\right)\left(\mathbf{v}+\mathbf{v}^{\star}\right)^{-1}(\mathbf{v}-1)
$$

after a few lines of careful non-commutative algebra. This is easily split up into components by means of the inversion formula $1 / \mathbf{v}=\overline{\mathbf{v}} /|\mathbf{v}|^{2}$; it agrees with the results of Stiefel and Waldvogel (1965) up to the sign of $v_{3}$. Both transformations regularize; the discrepancy is due to the different definition of the orientation in the inversions.

\section{References}

Birkhoff, G.D.: The restricted problem of three bodies. Rendiconti del Circolo Matematico di Palermo 39, 1 (1915); Reprinted in Collected Mathematical Papers, vol. 1. Dover Publications, New York (1968)

Celletti, A.: The Levi-Civita, KS and radial-inversion regularizing transformations. In: Benest, D., Frœschlé, C. (eds.), Singularities in Gravitational Systems, Lecture Notes in Physics, pp. 25-48. Springer-Verlag, Berlin, Heidelberg, New York (2002)

Hamilton, W.R.: On quaternions, or a new system of imaginaries in algebra. Philos. Mag. 25, 489-495 (1844)

Hopf, H.: Über die Abbildung der dreidimensionalen Sphäre auf die Kugelfläche. Math. Ann. 104 (1931); Reprinted in Selecta Heinz Hopf, 38-63. Springer-Verlag, Berlin, Heidelberg, New York (1964)

Kustaanheimo, P.: Spinor regularization of the Kepler motion. Ann. Univ. Turku, Ser. A 73, 1-7 (1964); Publ. Astr. Obs. Helsinki 102 
Kustaanheimo, P., Stiefel, E.L.: Perturbation theory of Kepler motion based on spinor regularization. J. Reine Angew. Math. 218, 204-219 (1965)

Levi-Civita, T.: Sur la régularisation du problème des trois corps. Acta Math. 42, 99-144 (1920)

Siegel, C.L., Moser, J.K.: Lectures on Celestial Mechanics, 290 pp. Springer-Verlag, Berlin, Heidelberg, New York (1971)

Stiefel, E.L., Scheifele, G.: Linear and Regular Celestial Mechanics, 301 pp. Springer-Verlag, Berlin, Heidelberg, New York (1971)

Stiefel, E.L., Waldvogel, J.: Généralisation de la régularisation de Birkhoff pour le mouvement du mobile dans l'espace à trois dimensions. C.R. Acad. Sc. Paris 260, 805 (1965)

Vivarelli, M.D.: The KS transformation revisited. Meccanica 29, 15-26 (1994)

Vrbik, J.: Celestial mechanics via quaternions. Can. J. Phys. 72, 141-146 (1994)

Vrbik, J.: Perturbed Kepler problem in quaternionic form. J. Phys. A 28, 193-198 (1995)

Waldvogel, J.: Die Verallgemeinerung der Birkhoff-Regularisierung für das räumliche Dreikörperproblem. Bull. Astronomique, Série 3, Tome II, Fasc. 2, 295-341 (1967a)

Waldvogel, J.: The restricted elliptic three-body problem. In: Stiefel, E., Rössler, M., Waldvogel, J., Burdet, C.A. (eds.), Methods of Regularization for Computing Orbits in Celestial Mechanics. NASA Contractor Report NASA CR 769, pp. 88-115 (1967b)

Waldvogel, J.: Order and chaos in satellite encounters. In: Steves, B.A., Maciejewski, A.J., Hendry, M. (eds.), Chaotic Worlds: From Order to Disorder in Gravitational N-Body Dynamical Systems, pp. 233-254. Springer, Dordrecht (2006) 